Ce texte est paru sous la référence :

Vailly, J., 2011, Qualité de vie ou vie de qualité ? Dépister une maladie génétique, in De la vie biologique à la vie sociale. Approches sociologiques et anthropologiques (J. Vailly, J. Kehr et J. Niewöhner Dir.), La Découverte, Paris, 161-189.

\title{
Qualité de vie ou vie de qualité ? Dépister une maladie génétique
}

\author{
Joëlle Vailly
}

Le mouvement historique d'intégration de la santé et de la vie aux stratégies politiques pointé par Michel Foucault [2001, p. 16-17] est relié à la notion de bien-être : «L'apparition d'une politique de santé doit être rapportée aussi à un processus beaucoup plus général : celui qui a fait du «bien-être » de la société l'un des objectifs essentiels du pouvoir politique. [...] [II] apparaît au XVIII ${ }^{\mathrm{e}}$ siècle une fonction nouvelle : l'aménagement de la société comme milieu de bien-être physique, de santé optimale et de longévité. », dit-ili ${ }^{1}$. C'est également dans la même lignée qu'il faut comprendre le concept de «qualité de vie » qui imprègne aujourd'hui de plus en plus de discours et de pratiques. Les instruments de mesure de la qualité de vie tiennent en effet leur existence de l'idée qu'il est possible de discriminer empiriquement les politiques qui accroissent le plus le bien-être général et collectif [Leplège, 2004]. Selon leurs promoteurs, ces mesures de qualité de vie présenteraient l'avantage d'allier une perspective quantitative, conçue comme objective (les critères de santé, de niveau de vie, de supports familiaux et affectifs, etc.), à une perspective subjective (l'évaluation qu'en fait la personne, le soignant, etc.). Dans ce cadre, des sociologues s'intéressent aux transformations du lien entre symptômes et maladie que les indicateurs de qualité de vie induisent [Armstrong, Lilford, Ogden, Wessely, 2007]. Ils montrent la façon dont l'introduction de mesures des activités de la vie quotidienne (activities of daily living, ADL) dans les questionnaires de santé à partir des années 1960 a introduit un décentrage vis-à-vis des manifestations immédiates de la pathologie, en y intégrant des effets plus en aval. A titre d'exemple, la façon dont l'arthrite peut se manifester non seulement par de la douleur, mais aussi par une difficulté à monter des escaliers a été prise en compte. Ce faisant, ces mesures ont été reliées à des perturbations des activités sociales, en parallèle avec la définition élargie de la santé que l'OMS développait, à savoir « un état de complet bien-être physique, psychologique et social, et pas seulement

\footnotetext{
${ }^{1}$ Ce travail a bénéficié du soutien du Gis-Institut des Maladies Rares. J'adresse mes remerciements à mes collègues de l'Iris, ainsi qu'aux professionnels de santé et aux familles de malades qui ont bien voulu se prêter à mes questions et à mes observations.
} 
comme l'absence de maladie ${ }^{2} »$. C'est à partir de là qu'ont pu se constituer les instruments de mesure de la qualité de vie, fondés essentiellement sur quatre types de paramètres : les symptômes, la santé mentale, les ADL et les activités sociales. Ainsi ces indicateurs combinent-ils la vie biologique (les symptômes) à certains aspects de la vie sociale (rencontrer ses amis, etc.).

Aborder la question de la vie et de sa qualité conduit bien sûr à s'intéresser de plus près au quotidien des malades, en particulier ceux atteints de maladies s'inscrivant dans la durée. Une littérature de sciences sociales abondante sur le fait de vivre avec une maladie chronique a d'ailleurs été accumulée dès les années 1970 [Strauss, Corbin, Fagerhaugh, Glaser, 1984]. Sont montrées en particulier les négociations et les interactions entre les différents acteurs impliqués, l'organisation des règles de la vie sociale dans la durée de la maladie et les transformations de cette organisation [Baszanger, 1986]. Dans cet ordre d'idée, des chercheurs étudient la façon dont le corps peut être intégré à la dimension sociologique de l'expérience de la maladie chronique d'une manière qui reconnaît à la fois les faits biologiques et sociaux : les faits biologiques peuvent devenir sociaux notamment lorsque d'autres personnes répondent au malade du point de vue de ses atteintes corporelles [Kelly et Field, 1996]. Par ailleurs, c'est l'attention à la maladie, à ses symptômes et aux atteintes qu'elle entraine, et ses relations avec la vie sociale qui intéressent d'autres auteurs [Timmermans et Haas, 2008]. Ces derniers considèrent qu'après avoir été trop proche des médecins dans les années 1950, en voulant prendre ses distances à leur égard, la sociologie a fini par délaisser la part physiologique de la maladie (sa gravité, ses manifestations, etc.) dans ses objets de recherche. En outre, les auteurs de l'article reprochent aux chercheurs en sciences sociales leur propension à lisser les différences entre maladies chroniques. Une autre étude vient indirectement à l'appui de cette idée, en s'attachant à analyser les expériences et les discours des malades atteints de maladies génétiques par comparaison aux maladies non génétiques [Petersen, 2006]. Du fait de leur caractère héréditaire, les premières suscitent des propos relatifs aux vies des membres de la famille affectés ou potentiellement affectés (la descendance) par la maladie. Des enjeux liés au partage d'information ou non sur la maladie entre ces membres, ou des préoccupations sur la santé ou le bien-être d'un futur enfant, peuvent alors émerger.

En lien avec ce dernier aspect, la recherche non seulement d'optimisation de la vie, mais aussi de mesure aussi précise que possible de sa qualité peut poser la question d'un renforcement des jugements de valeur sur les vies. Ces jugements affleurent déjà parmi toutes les techniques biomédicales qui rendent nécessaire une évaluation de la « qualité de vie », au sens précédemment défini, afin de prendre une décision informée pour l'action. On en voudra pour preuves les débats scientifiques et sociaux autour des choix qui doivent s'opérer en matière d'euthanasie, de réanimation néonatale, d'amniocentèse ou de diagnostic prénatal (avant la naissance). C'est pourquoi des sociologues et des anthropologues pointent que, derrière les pratiques de diagnostics pré-

${ }^{2} \mathrm{Cf}$. le site Internet de l'OMS : http://www.who.int/en/ 
natals notamment, est posée la question de savoir quelle vie vaut d'être vécue [Landsman, 2003 ; Press et Browner, 1997]. Même si des pratiques d'abandon des nouveau-nés frappés de maladies ou d'infirmités par les mères, lorsqu'ils sont considérés comme indignes de vivre, existent de longue date dans divers pays du monde [Landsman, 2003], un exemple significatif du poids croissant de la biomédecine en la matière est suggéré par les pratiques de fertilisation in vitro et de diagnostic préimplantatoire $^{3}$ en Grande-Bretagne. Celles-ci sont soumises en effet à une loi stipulant qu'une évaluation du bien-être des futurs enfants doit être effectuée en amont par l'unité médicale, sur la base de critères médicaux et sociaux [Ehrich, Williams, Scott, Sandall et coll., 2006]. Dans cette étude, l'équipe soignante considère d'un côté que son action doit être socialement et professionnellement responsable, d'un autre côté que cette évaluation du bien-être futur de l'enfant est intrusive et discriminatoire pour les couples concernés par rapport aux couples lambda. Selon les auteurs, cette tension peut être comprise comme un déplacement au moins partiel entre les solutions aux handicaps basées sur le social (le bien-être social) vers celles basées sur la génétique (le bien-être génétique). Cette étude présente l'intérêt de mettre le doigt sur des jugements de valeur en actes sur les vies futures et de préciser les critères de ces jugements. Du côté des éthiciens, certains d'entre eux s'alarment de la généralisation de certaines pratiques de diagnostic prénatal qui rendraient aujourd'hui des personnalités au talent immense, comme Mozart ou Einstein, « indignes de vivre ${ }^{4}$ ». D’un autre côté, comme l'indique le sociologue Nikolas Rose ${ }^{5}$, « le fait de chercher à éliminer un gène défectueux ne signifie pas que ceux qui sont nés avec ce gène ont des vies qui valent moins d'être vécues ou qu'ils méritent moins de soins et de soutien. Il n'y a pas d'élément permettant de penser que les parents qui ont des enfants porteurs de ces maladies pensent que leur vie est sans valeur ou leur accordent moins de valeur ». Le rapprochement de ces deux positions permet de souligner qu'avant et après la naissance, la question des jugements de valeur sur des vies potentielles ou des vies réelles peut se poser différemment. Si beaucoup de chercheurs en sciences sociales étudient la naissance d'enfants gravement malades ou handicapés en termes d'hypothèses dans le cadre de la grossesse et du conseil génétique [Remennick, 2006 ; Press et Browner, 1997], c'est un changement de perspective qui est ici proposé, en abordant la question de l'interprétation qui est faite des vies des malades une fois nés.

\section{Présentation de l'étude}

Il apparaît d'ores et déjà que ce qui est en jeu dans toutes ces questions intègre, mais va plus loin que la « qualité de vie ». En opérant un lien avec le diagnostic prénatal, ce qui est en jeu en effet inclut à la fois le vivant, l'existence et finalement la vie elle-

\footnotetext{
${ }^{3}$ Cette technique consiste à opérer une fertilisation in vitro, à prélever une cellule sur les embryons pour procéder à un test génétique et à implanter chez la femme les embryons choisis.

${ }^{4}$ Cf. l'article intitulé « La France au risque de l'eugénisme » (Le Monde du 4-5.2.07).

${ }^{5} \mathrm{Cf}$. http://www.abc.net.au/rn/talks/bbing/stories/s1539272.htm. C'est moi qui traduis.
} 
même. C'est pourquoi je propose de renverser le concept de qualité de vie pour parler de celui de « vie de qualité », qui semble au cœur des pratiques. Le moyen d'étude ici mobilisé pour aborder ce passage entre qualité de vie et vie de qualité consiste à analyser la façon dont s'opère sur le terrain l'articulation entre la recherche de la bonne santé (par les soins aux malades), moralement consensuelle, et l'élimination de la mauvaise santé (par le diagnostic sur les fœetus), parfois plus controversée. Saisir cela peut contribuer à une meilleure compréhension des développements de la biomédecine et de l'espace dans lequel elle se situe. L'hypothèse réside dans le fait que la naissance est un moment privilégié pour cette analyse.

Plus précisément, cette recherche est partie prenante d'une étude des enjeux scientifiques, politiques que moraux du dépistage néonatal de la mucoviscidose (DNM) ${ }^{6}$. Ce dépistage, qui consiste à dépister tous les nouveau-nés pour cette maladie génétique, a été lancé en 2002 en France. Il s'inscrit dans le contexte d'un développement quantitatif substantiel des dépistages néonatals, en Europe et aux Etats-Unis notamment : une trentaine de maladies génétiques rares est dépistée à la naissance dans les différents Etats des Etats-Unis (en moyenne), quinze en Allemagne, cinq en France, etc. Le texte présenté ici traite donc de la façon dont est poursuivi l'objectif de garder une vie de qualité à travers les pratiques de DNM. Dans une première partie, seront analysés les efforts de soins à l'égard des enfants malades pour atteindre cet objectif. Comment cette vie de qualité est-elle conçue par les médecins et les familles de malades ? Dans ce cadre, comment les vies biologiques et les vies sociales sont-elles liées ou coproduites ? J'en viendrai ensuite à la façon dont est gérée sur le terrain la relation entre les approches néonatale (après la naissance) et prénatale (avant la naissance). Au-delà des différentes pratiques professionnelles, je montrerai en quoi elles sont liées ou en tension, et comment globalement elles convergent. On verra également comment l'idée de précocité, et plus largement la question des temporalités, agissent comme un opérateur de ce lien. Enfin, sera proposée une discussion plus générale sur cette notion de vie de qualité autour de la naissance.

Au plan des pratiques, précisons que les dépistages néonatals en France sont proposés et effectués sous la responsabilité d'une association professionnelle, appelée Association française pour le dépistage et la prévention des handicaps de l'enfant (AFDPHE), rassemblant surtout des pédiatres et, dans une moindre mesure, des généticiens. Comme cela a été montré dans un article précédent, la condition principale à la décision du DNM, effectué sous l'égide de l'AFDPHE donc, a été la création de centres de soins spécialisés pour la maladie, afin d'assurer une prise en charge adaptée dès le diagnostic établi [Vailly, 2006]. Une cinquantaine de centres de soins spécialisés pour la mucoviscidose, appelés Centres de ressource et de compétence de la mucoviscidose (CRCM), situés pour la plupart au sein de centres hospitalo-universitaires, ont

\footnotetext{
${ }^{6}$ Rappelons que, par définition, le dépistage consiste à identifier les personnes, au sein d'une population, qui risquent d'être affectées par une maladie (auquel cas le dépistage est suivi d'un test diagnostic de confirmation) ou en sont déjà affectées sans le savoir. La mucoviscidose est une maladie rare qui se traduit par des problèmes respiratoires et digestifs souvent graves. Elle touche environ 6000 malades en tout et 180 nouveau-nés par an en France. Il n'existe pas de traitement curatif de la maladie et l'espérance de vie des malades est d'environ 40 ans en Europe.
} 
ainsi été créés lors du lancement du dépistage. Ceux-ci sont des centres multidisciplinaires rassemblant des pédiatres, des généticiens, des kinésithérapeutes, etc.

Cette recherche s'appuie d'abord, au plan local, sur une enquête de terrain menée en 2005, pendant sept mois, dans un CRCM situé au sein d'un grand hôpital de la région parisienne. Elle repose sur des observations de consultations de pédiatrie spécialisées dans la mucoviscidose (91 observations, dont un tiers avec des enfants dépistés) et sur des entretiens avec les cliniciens et les mères des jeunes enfants dépistés à la naissance (22 entretiens). Les entretiens avec les professionnels portent notamment sur leurs pratiques, en mettant l'accent sur ce que le DNM change en la matière, sur le bilan qu'ils font de ce dépistage, ainsi que sur les malades qu'ils suivent. Avec les mères des dépistés, les entretiens consistent à retracer en détail chaque étape du dépistage néonatal et du suivi associé. Ils permettent également de connaître le bilan qu'elles font du dépistage et certains aspects sensibles liés à la valeur des vies de malades. Lorsque les pères directement impliqués dans le suivi du malade sont présents et souhaitent assister à l'entretien, ils sont inclus à la recherche. Cette recherche s'appuie ensuite, au plan national, sur l'observation de réunions biomédicales nationales sur le DNM effectuées en 2003-2005 (16 observations), ainsi que sur des entretiens avec les principaux acteurs impliqués dans le processus de décision du DNM au plan national (23 entretiens), et sur l'étude de documents (plaquettes d'information sur les dépistages néonatals, statistiques nationales, etc.).

\section{La vie des enfants dépistés malades}

La thématique de la qualité de vie est invoquée dans la plupart des documents qui arguent en faveur du DNM et d'une prise en charge précoce. Telle plaquette d'information sur les dépistages néonatals distribuée aux parents en maternité précise ainsi qu' " une prise en charge précoce et rigoureuse [...] assure au malade une meilleure qualité de vie, malgré l'absence d'un traitement spécifique conduisant à la guérison définitive du malade » [AFDPHE, 2001]. Tel communiqué de presse envoyé par les organisateurs du dépistage au moment de son lancement insiste sur le fait qu' «il est important de dépister [la mucoviscidose] au plus tôt, pour améliorer la qualité de vie des enfants dépistés et prolonger leur espérance de vie »[Assurance Maladie, 2000]. Et l'on pourrait relever d'autres exemples. C'est dire qu'une prise en charge précoce et appropriée est gage pour les médecins d'une vie aussi bonne que possible. A cette fin, les enfants dépistés malades sont envoyés dans les CRCM, et c'est ainsi qu'à partir du dépistage, toute une prise en charge est mise en place dès le plus jeune âge.

La surveillance approfondie s'opère à travers les consultations (mensuelles les six premiers mois, puis bimensuelles), avec examen bactériologique systématique des crachats, et les journées semestrielles en hôpital de jour. L'examen des crachats comporte ce double sens de l'examen, en tant qu'observation et en tant qu'épreuve. En effet, c'est lui qui livre le verdict : l'enfant «a du pyo » (il est infecté par un germe appelé 
pyocyanique) ou n'en a pas. Il peut également être infecté par d'autres germes, mais le plus redouté est bien le premier. Le bilan approfondi en hôpital de jour livre quant à lui toute une palette de résultats, au prix d'examens parfois intrusifs. Entre la surveillance et le soin, dès le diagnostic établi sur les nouveau-nés, viennent les séances de « kiné » (kinésithérapie respiratoire), en moyenne deux fois par semaine pour les formes légères et si l'enfant va bien, mais jusqu'à deux fois par jour s'il est « encombré ». Les séances de «kiné » peuvent facilement impressionner les non habitués, au vu des efforts déployés pour extraire les mucosités du plus profond des voies respiratoires des nourrissons. Lorsque ceux-ci grandissent, c'est surtout le caractère répétitif et fastidieux des séances souvent quotidiennes qui s'impose aux enfants et aux adolescents. Concernant les soins proprement dits, que le bilan médical de l'enfant ne soit pas satisfaisant et un véritable plan d'action est déclenché (régime alimentaire modifié, nouveaux antibiotiques, etc.). Que la situation se complique encore et la sémantique utilisée peut revêtir un caractère combatif ou guerrier : il s'agit de donner « un coup de poing au pyo » ou d'être « en vigilance armée ». Les armes ont pour noms des antibiotiques, des cures intraveineuses, des aérosols et des enzymes digestives avalées. Les alliés sont les régimes alimentaires riches, l'hygiène et des règles de vie qui évitent de s'exposer aux microbes. Ce combat représente au quotidien pour les petits enfants qui ont des formes plutôt sévères deux heures de soins spécifiques à la maladie.

En même temps, les pédiatres sont conscients qu'une vie de qualité pour l'enfant dépisté ne peut se résumer aux soins, notamment parce qu'il s'agit d'une maladie chronique. Comme l'écrit la sociologue Isabelle Baszanger [1986], « les malades [atteints de maladie chronique] doivent aller au-delà d'un travail de soins médicalement défini. Il leur faut aussi gérer les conséquences de la maladie sur leur organisation de vie [et] leurs rapports avec les autres ». Autrement dit, étant donné que la maladie s'inscrit dans la durée, elle ne peut être envisagée comme une suspension de l'accomplissement des rôles sociaux normaux. L'un des enjeux est le maintien, si possible, des insertions sociales, car un équilibre il faut garder entre la vie biologique (l'hygiène, les soins) et certains aspects de la vie sociale (les jeux, les sorties, etc.). En consultation, des exemples permettent de situer la zone d'équilibre, notamment lorsque les mères demandent au/à la pédiatre des précisions sur les loisirs auxquels les enfants malades peuvent avoir accès sans risquer d'aggraver la maladie. Elles cherchent à mettre en regard le bénéfice des loisirs (aller au parc, jouer dans la piscine, voir d'autres enfants, etc.) avec le risque perçu auxquels ceux-ci exposent. Certaines peuvent chercher à négocier, voire à exiger un moment de convivialité (par exemple faire participer son enfant à la fête de Noël de l'hôpital - sans se rendre compte que cela l'expose à des risques de contamination, comme le lui explique la pédiatre). La formule qui consiste à dire que l'enfant ne peut pas rester dans « une bulle » revient plusieurs fois lors des consultations ou des entretiens. L'équilibre entre normalisation et «dé-normalisation » de la vie en raison de la maladie peut être difficile à trouver [Strauss, Corbin, Fagerhaugh, Glaser et coll., 1984].

Plus généralement, il existe une tension entre le fait de préserver l'enfant des infections et des risques divers, et le fait de lui permettre une vie de qualité qui le fait - ou le 
ferait - entrer dans la catégorie de normalité. A ce titre, il est formellement déconseillé de placer les enfants en crèche en raison des risques d'infection, mais très encouragé de les envoyer à l'école. Dans le même esprit, lors d'assises nationales associant des professionnels et quelques représentants de familles de malades, un pédiatre et un pneumologue citent parmi les objectifs médicaux le fait de «concilier le suivi rapproché et la qualité de vie [...], de façon que nous ayons devant nous des enfants et non des mucoviscidoses » ou de «mettre en accord des mesures d'hygiène et une qualité de vie maintenue ». Un père d'enfant malade, responsable d'association de malade, souligne à son tour que «le combat commun [mené] ensemble a pour seul et unique objectif d'améliorer la qualité des soins en maintenant la qualité de vie ». Les deux éléments (suivi rapproché et qualité de vie) sont placés ici en tension, et il s'agit de trouver un mode de conciliation entre les deux. Or, l'équilibre à trouver peut être rendu plus complexe lorsque les loisirs et les soins sont tous deux à la fois porteurs de dangers potentiels et de bénéfices. La question des jouets est typique de cette dualité et peut faire l'objet de discussions entre les soignants. Celles-ci montrent que suivant l'environnement, les jouets peuvent contribuer à une bonne vie pour un enfant (les jouets à la maison contribuent à la «qualité de vie ») ou sources de dangers (les jouets à l'hôpital sont sources de germes). D'ailleurs, lors d'une observation de consultation, une pédiatre sort de la pièce un jouet de l'hôpital en soulignant que «c'est un vecteur de germes », ce qui n'empêche pas certains enfants dépistés malades de jouer avec dans la salle d'attente. En miroir, les soins peuvent à la fois être perçus comme des contraintes qui pèsent sur la vie de l'enfant et un atout qui lui apporte une meilleure santé donc, au total, une meilleure vie. Ainsi, lors de ces mêmes assises de soins, une pédiatre et un pneumologue disent-ils : «On a augmenté les soins ; ça aurait pu être au détriment de la qualité de vie des enfants et c'est relativement rare » et : «Une hygiène de base correcte induit une qualité de vie, une qualité des soins sous-tend une meilleure qualité de vie. » Dans cette conception, la tension entre soins et qualité de vie disparaît car les soins permettent une qualité de vie.

Au total, comment résumer cette situation complexe ? Les médecins et les parents sont conduits à des arbitrages parfois cruels entre vie biologique (préserver l'état de santé) et certains aspects de la vie sociale (voir des enfants, jouer, etc.). Mais ces arbitrages ne se limitent pas à une relation binaire, car la vie biologique influe en négatif sur la vie sociale (deux heures de soins quotidiens limitent les activités), mais aussi en positif (les soins permettent d'aller mieux donc de profiter de ces activités). De manière symétrique, la vie sociale influe en négatif sur la vie biologique (aller à la piscine apporte un risque d'infection), mais aussi en positif (jouer avec l'eau permet de garder sa joie de vivre, ce qui influe sur la santé). Cette intrication est liée à la prise en compte des activités sociales dans la notion de «qualité de vie » et au fait que ces activités sociales ont en retour des effets sur la mucoviscidose. Dans la conception des professionnels, le fait de garder une vie de qualité se situe principalement dans le registre de la vie biologique, dont ils sont gardiens, mais pas uniquement, puisque cela implique également de sortir, jouer, aller à l'école, etc. Leur conception intègre dans les pratiques cliniques les transformations induites par les instruments de qualité de vie 
tels qu'ils ont été présentés en introduction. En même temps, le lien avec les lésions corporelles reste plus fort qu'à travers l'analyse d'autres auteurs, qui évoquent une qualité de vie mesurée à partir «de symptômes flottant librement» et «sans lien » avec les lésions [Armstrong, Lilford, Ogden, Wessely, 2007]. Ceci n'est assurément pas transposable à notre cas, et cette différence s'explique par la nature de la maladie dont il est ici question, on ne peut plus inscrite dans les corps. A partir de cette intrication du social et du corporel, on mesure les dilemmes et les paradoxes que la mucoviscidose véhicule. A ce titre, dans un ouvrage autobiographique, une mère fait part de son effroi devant les premières séances de kinésithérapie respiratoire auxquelles elle assiste et elle ajoute : "Ces enfants sont maltraités et cette violence est nécessaire à leur survie. Quel triste paradoxe !» [Laurent-Seguin, 1999, p. 92]. Sans doute pourrait-on aller plus loin dans le paradoxe à propos des jeunes enfants et dire : «Ces enfants sont maltraités et cette violence est nécessaire à leur vie de qualité. » Et parfois effectivement à leur vie tout court.

En effet, au fil des années, les problèmes des jeunes malades se compliquent fréquemment. Peu à peu ils prennent conscience de la chronicité de leur maladie et, le plus souvent, leur traitement s'alourdit. L'état médical devient progressivement un enjeu vital au sens propre, qui interfère de plus en plus avec les activités (aller à l'école, partir en vacances, etc.). Ainsi au cours des observations, tel adolescent régurgite de l'acidité quand il tousse, il doit se faire opérer et va manquer l'école deux semaines, telle jeune fille dont les fonctions respiratoires sont mauvaises doit passer par une fibroscopie sous anesthésie générale, puis enchaîner avec une cure intraveineuse de quinze jours, tel jeune garçon a des problèmes digestifs douloureux, il manque souvent le lycée, il est nécessaire de procéder à une coloscopie et à des lavements importants, etc. Les cures et les hospitalisations deviennent plus fréquentes, en raison de la chronicisation de l'infection des poumons. Il arrive que les jeunes malades pleurent en consultation à l'annonce d'une nouvelle cure intraveineuse ou de traitements lourds. Pire, à ces moments, chacun - malade, parent et médecin - approche la dimension immanquablement tragique de la médecine : le fait que chacun soit mortel. Mais le tableau n'est pas toujours aussi morbide. A l'inverse, d'autres adolescents se portent bien et il est difficile d'imaginer qu'ils sont malades. Ils viennent à l'hôpital pour des contrôles, mais ne sont que peu affectés par la maladie et celle-ci est peu visible. Toutes les incertitudes sur les conséquences d'une même altération génétique (mutation) ou d'une même maladie, que l'on retrouvera plus loin, trouvent une traduction en termes de perspectives de vie et de maladie. Etant donné tout ce contexte, émerge la question de la valeur d'une vie où les soins tiennent souvent tant de place. Et d'abord, concrètement, pour les familles et les médecins, de ce qu'il convient de faire lors d'autres grossesses à venir.

\section{Quand le dépistage néonatal reconfigure le diagnostic prénatal}


L'enquête à l'hôpital montre que le dépistage néonatal peut être relié à la question du diagnostic prénatal à différents titres. D'abord, il faut savoir qu'avant la mise en place du DNM, lorsque deux enfants naissaient à intervalle rapproché dans une famille, il pouvait survenir la naissance d'un deuxième enfant malade, alors que le diagnostic n'avait pas encore été posé sur le premier. Or aujourd'hui, le dépistage néonatal permet de poser un diagnostic précocement sur le premier enfant, de proposer un diagnostic prénatal lors d'une deuxième grossesse et donc d'éviter les fratries de plusieurs enfants atteints de mucoviscidose. Cet argument, tout en n'étant pas central, est d'ailleurs avancé en tant que justification au DNM par certains pédiatres et biologistes [Vailly, 2004]. Lorsque les parents souhaitent un autre enfant, le pédiatre les encourage à rencontrer le généticien en vue d'un conseil génétique et d'un éventuel diagnostic prénatal. Une large majorité de femmes en France - plus de 80\% - procèdent à une interruption de grossesse en cas de mucoviscidose détectée chez le fœetus [Agence de la biomédecine, 2007]. Ceci constitue donc un premier point d'articulation entre néonatal et prénatal, qui fait l'objet de peu de débats chez les professionnels, tant la lourdeur des soins pour un premier enfant malade peut être grande pour les malades et leur famille. Les autres points d'articulation seront davantage détaillés ici.

Le deuxième point d'articulation est lié à l'extension de l'idée d'anormalité médicale, un processus au demeurant plus général de la biomédecine [Vailly, 2008]. Pour des raisons techniques, environ $15 \%$ des nouveau-nés dépistés et considérés comme atteints sont en fait porteurs de formes dites frontières, ni tout à fait normales, ni tout à fait pathologiques. Ils peuvent par exemple être porteurs de mutations conduisant soit à une mucoviscidose classique, soit à des affections du pancréas, soit à une forme d'infécondité masculine. Une partie d'entre eux n'auraient probablement pas été détectée sur symptômes ou l'auraient été bien des années plus tard, ce qui traduit une forme de sur-diagnostic à la naissance avec le DNM. Ceci connaît des prolongements cliniques, puisque les pratiques des pédiatres consistent dans une large majorité des cas à suivre les enfants porteurs de formes frontières, y compris de façon assouplie, plutôt que de les considérer comme sains [Vailly, 2008]. C'est dire que tout en s'interrogeant sur leurs propres pratiques et sur les conséquences de la catégorisation en enfant mucoviscidosique, ils témoignent d'une certaine anxiété vis-à-vis d'une exclusion inappropriée de mucoviscidose. De plus, comme cela a été détaillé précédemment [Vailly, 2008], dans le centre où l'enquête a été effectuée, lorsqu'une mutation repérée lors du dépistage néonatal est peu connue ou associée, dans la très grande majorité des cas, à une forme bénigne et, dans quelques cas, à une forme classique, le diagnostic prénatal sur une grossesse ultérieure est proposé. Seules les situations où l'on peut affirmer que le profil génétique mènera dans tous les cas uniquement à une forme d'infécondité sont exclues du diagnostic prénatal, les professionnels considérant alors que l'interruption de grossesse pour raison médicale n'a pas lieu d'être. En somme, lorsqu'une forme frontière est identifiée lors du dépistage néonatal, le diagnostic prénatal est proposé lors d'une grossesse ultérieure dans la famille, montrant la façon dont la norme sur les enfants à naître, cette fois, est modifiée. Les formes frontières dessinent donc une nouvelle articulation entre dépistage néonatal et diagnostic prénatal fondée 
sur une extension de l'idée d'anormalité et une forme de passage à la norme.

Les individus dits « hétérozygotes », autrement dit porteurs d'une mutation, donc non malades dans le cas de la mucoviscidose (il en faut deux pour être atteint), constituent une autre articulation. Pour des raisons techniques déjà décrites [Vailly, 2008], le DNM amène à identifier environ 2\% (soit 400 à 500 enfants) de la population des nouveau-nés hétérozygotes naissant chaque année en France. Dès lors, un enfant dépisté à la naissance qui se révèle hétérozygote pourra demander un test génétique plus tard lorsqu'il ou elle voudra un enfant. De même, ses apparentés (oncle et tantes, cousins, etc.) sont-ils incités à rencontrer un généticien de façon à pouvoir bénéficier d'un diagnostic prénatal éventuel si nécessaire. Le fait de repérer des hétérozygotes au cours du dépistage néonatal, même en nombre limité, induit donc la possibilité de nouveaux diagnostics prénatals à plus ou moins longue échéance. Pour certains médecins, cette pratique est perçue positivement, puisqu'elle pourrait permettre de repérer un nombre croissant d'hétérozygotes dans la population générale, de proposer de plus en plus de diagnostics prénatals et, au total, d'éviter des souffrances. Mais, la plupart du temps, cette identification d'hétérozygotes est perçue négativement par eux, pour plusieurs raisons. En premier lieu, c'est l'objectif, voire le sens même qu'ils donnent à leurs propres actions, qui est en jeu. Dépister un hétérozygote, ce n'est pas identifier un malade pour lui apporter des soins, c'est repérer une caractéristique génétique pouvant déboucher sur un test prénatal. Ce changement risque de brouiller la finalité de l'action et de déplacer les soignants de la sphère thérapeutique vers une sphère selon eux plus obscure et détachée de la cible initiale. En outre, au-delà des normes de pratiques, l'examen des caractéristiques génétiques des enfants mineurs et le rapport à la loi qui l'encadre se trouvent modifiés. En effet, celle-ci précise que cet examen ne peut être prescrit chez un enfant mineur que si ce dernier ou sa famille peuvent personnellement bénéficier «de mesures préventives ou curatives immédiates ${ }^{7}$ ». Or, le bébé hétérozygote ne bénéficie pas de telles mesures puisqu'il n'est pas malade. Pour cette raison, certains pédiatres et généticiens insistent sur le fait que le DNM a «bouleversé l'examen des caractéristiques génétiques des mineurs ». En deuxième lieu, ceux-ci peuvent questionner l'étendue réelle ou redoutée de l'action à laquelle eux-mêmes participent. Il se profile parfois chez eux la crainte, de proche en proche, d'un dépistage de masse ou d'un « eugénisme ». Selon ce point de vue, le fait de repérer des hétérozygotes à la naissance et d'en informer les familles pourrait conduire en effet à identifier un nombre croissant d'hétérozygotes, même si le recul manque encore à l'heure actuelle pour jauger la diffusion effective de cette information au sein des familles. Cette inquiétude est parfois renforcée par une surestimation du nombre d'hétérozygotes identifiés. En troisième lieu, les conséquences imprévues de l'action engagée peuvent susciter une inquiétude, liée au fait que des enfants hétérozygotes porteurs d'une mutation classée comme a priori peu sévère, mais susceptibles de demander un conseil génétique à l'âge adulte, puissent être identifiés. Revient ici en dé-

\footnotetext{
${ }^{7}$ Il s'agit du décret n²000-570 du 23 juin 2000. En réalité, la loi est frappée d'ambiguité, car un dépistage d'hétérozygote, dans la mesure où il débouche sur un conseil génétique, pourrait être considéré comme une forme de «mesure préventive pour la famille».
} 
bat la question des mutations impliquées dans des formes frontières de la maladie, traitée précédemment.

Au total, ces trois éléments, diagnostic prénatal pour éviter les fratries de malades, formes frontières et repérage d'hétérozygotes constituent les trois premiers modes d'articulation entre le DNM et le diagnostic prénatal. Ils restent dans le cadre des grossesses dites «à risque », lorsqu'une mutation plus ou moins sévère est déjà identifiée dans une famille. D'une part, ils sont ancrés dans les procédures techniques de dépistage néonatal, d'autre part, ils touchent à ce que Mitchell Dean [1999] appelle les «pratiques éthiques », autrement dit la finalité de l'action dans un cadre éthique. En effet, tout en passant par un dépistage visant à une prise en charge précoce, les acteurs biomédicaux sont parfois embarrassés, car leur attention est susceptible selon eux de se décentrer de l'enfant vivant et souffrant vers des enfants asymptomatiques ou vers la descendance du futur adulte et de sa famille. Cela peut induire des interrogations de leur part, aussi bien pour les formes frontières que pour les hétérozygotes.

\section{Le temps gagné}

Un autre aspect par lequel le dépistage néonatal interfère avec le diagnostic prénatal est lié au fait que le premier fait bouger les esprits vis-à-vis du second. Plus exactement, il fait bouger les esprits sur le dépistage de masse des femmes enceintes. Comme cela a été suggéré plus haut à travers les extraits de documents cités, garder une vie de qualité grâce au DNM consiste surtout à repérer la maladie et à prendre en charge de manière adéquate tôt , sachant tout de même qu'avant la mise en place du dépistage, les deux tiers des enfants étaient diagnostiqués avant l'âge d'un an [ONM, 2002]. Certains cliniciens expliquent en entretien que des malades diagnostiqués à l'âge de 6 ou 7 ans peuvent voir leur santé déjà bien dégradée, faute d'une connaissance suffisante de la maladie parmi les pédiatres non spécialisés et d'un suivi adéquat. Cet argument temporel connaît des prolongements. Pour bien les comprendre, il est utile de rappeler qu'en France, il n'existe pas de limite légale dans le temps à l'interruption médicale de grossesse, puisque celle-ci peut être effectuée pratiquement jusqu'à terme. Il convient de souligner également que les mères et les parents concernés par cette enquête sont parents d'enfants dépistés âgés de moins de deux ans.

A plusieurs reprises au cours de l'enquête à l'hôpital, des médecins expliquent en entretien que certains parents, suite à un dépistage positif de leur enfant à la naissance, les interrogent sur les raisons pour lesquelles le test n'est pas effectué pendant la grossesse. Cette situation ne semble pas spécifique du centre où l'enquête de terrain a été effectuée puisque, au cours de réunions ou d'entretiens, des généticiens issus d'autres régions de France mentionnent le même type de propos. Aux dires des professionnels, le lien entre dépistages néonatal et prénatal est établi par les parents selon différents modes : une remontée dans le temps (« Pourquoi on n'a pas fait le test avant ?»), une convergence entre les techniques mobilisées pour les deux dépistages («Mais techniquement, est-ce que c'est possible ? ») et une combinaison des deux éléments précé- 
dents («Pourquoi on ne cherche pas la maladie avant, puisqu'on peut le faire après ? »). Les entretiens effectués auprès des mères ou des parents d'enfants dépistés corroborent d'ailleurs les propos des médecins, pour une proportion substantielle de familles, en particulier chez les mères ou les parents d'enfant dépisté malade (plus de la moitié). Un point de méthode qu'il faut souligner est que ces interrogations apparaissent dans tous les cas spontanément, sans allusion préalable de l'enquêtrice au dépistage prénatal. Elles sont formulées parfois même en tout début de conversation. Lors de ces entretiens, il se dégage de nouveau la thématique du temps, avec des propos du type : «On a perdu beaucoup de temps », «C'est dommage de ne pas le faire avant », «Je sais pas pourquoi on ne l'a pas fait avant », etc. Tout se passe comme si la recherche de précocité qui imprègne toute la démarche du dépistage néonatal se prolongeait par une progression en amont vers la période prénatale. Ensuite, à partir du moment où un dépistage concernant tous les fotus est promu, s'exprime en lien avec celui-ci la problématique du type de vie vécue ou à venir pour l'enfant, ainsi que son évaluation. Les formules suivantes l'attestent : «Le mode de vie est particulier, c'est un peu lourd », «C'est pas facile tous les jours », «C'est toute une vie qu'il faut le prendre en charge », «C'est un adulte qui va déjà avoir un poids à porter plus lourd qu'un autre. » Finalement, l'une des mères d'un très jeune enfant conclut : «L'enfant, lui, n'est pas heureux. » Une autre explique qu'elle « ne fera pas subir ça » à son nouvel enfant. Bien sûr, cette problématique entre en conflit avec l'attachement et l'amour des mères et des parents pour leur enfant déjà né. A plusieurs reprises, elles et ils diront au même moment de l'échange : « Je ne regrette pas d'avoir mon fils », « Aujourd'hui il est là, je l'aime, c'est mon enfant », «S'il était à refaire [en désignant son enfant malade], je le referais celui-là », etc. Dans un cas, le père ne peut d'ailleurs formuler ce qui lui paraît probablement la négation de l'existence de sa fille, qu'il soigne avec tant d'attention, et il dit simplement : « Si on nous avait dit : 'Votre enfant a la mucoviscidose'... Je sais pas... » Finalement, c'est son épouse qui exprime leur souhait commun d'un dépistage prénatal généralisé. La valeur de l'enfant est alors parfois réaffirmée au même moment au cours de l'entretien, comme dans le cas de cette mère qui ne peut s'empêcher d'ajouter au sujet de son fils malade: "C'est un sacré bonhomme quand même. » Enfin, les parents mettent parfois en relation les difficultés des enfants malades et de leurs parents avec l'environnement socioéconomique de la famille et les politiques sociales. Ils mettent alors l'accent sur le manque d'aide en matière de maladie ou de handicap, et sur le coût de la maladie pour les parents ou pour la société. Bien sûr, cela ne signifie pas néanmoins que les familles les plus précaires seraient davantage favorables au dépistage prénatal ou pratiqueraient davantage l'interruption de grossesse.

Si le DNM est marqué par un grand souci de précocité, il n'est pas étonnant qu'une proportion substantielle de parents d'enfants dépistés poursuivent cette logique et s'étonnent d'un dépistage néonatal jugé encore trop tardif. Cette idée pourrait être renforcée par la préconisation, formulée notamment par certains professionnels, d'une information sur les dépistages néonatals pendant la grossesse. Si l'information est donnée avant la naissance et si la technique de repérage de la maladie paraît dispo- 
nible, pourquoi attendre ? Mais les mères vues en entretiens expriment le point de vue de celles avec des enfants malades une fois nés. Elles se trouvent dans une position paradoxale, car tout à la fois elles combattent la maladie, aiment l'enfant qui incarne cette maladie et sont amenées à qualifier sa vie. Dans le même ordre d'idée, l'anthropologue Gail Landsman [2003] explique que les mères d'enfants handicapés naviguent entre différents discours et se trouvent au centre d'un paradoxe, disant à la fois de leur enfant qu'elles l'aiment tel qu'il est et qu'elles feraient n'importe quoi pour le changer (enlever le handicap). Il est possible que les points de vue des mères dans le cas de la mucoviscidose évoluent au fur et à mesure que l'enfant grandit, une question qui relèverait d'une autre enquête. A l'appui de cette idée, les mères qui viennent juste de recevoir le diagnostic de handicap, dit Gail Landsman [2003], adoptent un discours centré sur le « retard de développement» (impliquant qu'il y aura un rattrapage possible), alors que plus tard elles adoptent un discours assumé autour du «handicap » (sans rattrapage): lorsque ces mères comprennent que leur enfant n'atteindra probablement jamais la conception de ce qu'est un enfant «normal», la plupart réévalue le sens et la valeur de la normalité, et développe la critique d'un discourt qui diminuerait la valeur de leur enfant. D'autre part, il convient de souligner que les mères des enfants atteints de mucoviscidose suivies au cours de l'enquête reproduisent une logique des médecins français bien ancienne, selon laquelle il n'y a bien souvent pas eu de hiatus entre l'action précédant et celle suivant la procréation. Dès le XVII ${ }^{e}$ siècle, la «callipédie » a entendu se consacrer aux soins autour de la grossesse et de l'accouchement, mais aussi de l'éducation de l'enfant dans ses premières années [Carol, 1995]. A la fin du XIX ${ }^{\mathrm{e}}$ siècle, à travers des disciplines médicales telles que la puériculture et la vénérologie, l'expérience médicale des pédiatres les a conduits à remonter progressivement jusqu'aux ascendants pour expliquer, puis pour combattre certaines pathologies [Carol, 1995]. Un faisceau d'indices argue donc historiquement en faveur d'un tel phénomène de remontée en amont, qui rejoint celui que l'on observe aujourd'hui sur le terrain. Mais qu'en disent les médecins ?

Au cours de l'enquête à l'hôpital, aucun des pédiatres et des généticiens rencontrés n'a plaidé pour un dépistage de la mucoviscidose chez toutes les femmes pendant la grossesse. L'un des généticiens s'interroge toutefois en entretien, au vu des sommes d'argent mobilisées pour le dépistage néonatal, sur le fait de savoir « s'il n'y aurait pas eu intérêt à faire un dépistage pendant les grossesses », tout en ajoutant que cela aurait eu «un côté eugéniste, pas eugéniste, mais un peu dérangeant d'empêcher tous ces enfants de naître ». Lors d'une consultation, le même généticien dit incidemment à des parents : «On fait un dépistage à la naissance ; ça valait peut-être mieux de faire en prénatal, mais c'est comme ça. » Est-ce à dire que le dépistage néonatal peut faire bouger les esprits sur le dépistage prénatal chez les professionnels également ? En partie sans doute, et d'autres données à ce propos sont présentées plus loin. Mais l'association éventuelle avec l'eugénisme ou une idée apparentée bride les velléités de généralisation. Dès lors, cette idée devient difficilement défendable, ou même dicible, en raison des valeurs morales. Même s'ils ne connaissent pas tous l'histoire de l'eugénisme dans ses détails, les professionnels baignent dans un contexte qui ne peut 
s'abstraire d'une certaine distanciation à son égard. L'histoire de l'eugénisme en France est éclairante, car elle est fondée sur une double originalité : l'eugénisme y a été porté par des médecins et non, comme dans d'autre pays, par des statisticiens ou des biologistes [Carol, 1995]. Ceci explique en partie pourquoi il a été peu développé en France. En effet, au plan moral, l'eugénisme le plus dur - l'eugénisme d'exclusion des malades - s'opposait à la déontologie et aux convictions personnelles des cliniciens. Au plan professionnel, l'eugénisme d'Etat allait à l'encontre de leur pratique libérale, mettant en péril leur liberté d'action, leur prestige et leurs revenus [Carol, 1995]. Quant à l'eugénisme familial et privé, il n'a connu que peu de débouchés. Rejoignant d'autres études qui pointent l'importance du contexte sociohistorique pour saisir ces questions [Beck et Niewöhner, 2009], soulignons que la relativement faible implantation historique de l'eugénisme en France permet en partie de comprendre dans quel contexte il reste un sujet sensible à même de forger un ethos.

Les professionnels évoquent alors une forme de dépistage prénatal non généralisé, mais banalisé, par un autre biais : celui de la mise au point d'une technique de repérage de mutations sur les cellules foetales circulant dans le sang maternel dès les débuts de la grossesse. Cette technique, maintenant mise en œuvre de façon expérimentale, permet par une simple prise de sang chez les femmes enceintes de repérer des mutations et de proposer très tôt une interruption de grossesse. Elle rejoint ainsi la problématique de la précocité, puisqu'elle vise à éviter aux femmes des interruptions de grossesse tardives, dont le caractère traumatisant est souligné par les généticiens. En outre, cette nouvelle technique évite les risques de fausses-couches, toujours présents lors des techniques classiques telles que les biopsies de trophoblaste ou les prélèvements de liquide amniotique. Des généticiens sont convaincus qu'un nombre croissant de femmes fera appel à cette nouvelle technique. Au total, une fois le seuil de la naissance franchi, le même souci de précocité imprègne ainsi le diagnostic prénatal et trouve une traduction dans ces innovations. Comme l'écrit l'anthropologue Patricia Kaufert [2000, p. 176], «la tentation, particulièrement pour les cliniciens, est de remonter continuellement les limites et de «trouver » la maladie à des stades de plus en plus précoces ${ }^{8}$. On ne saurait mieux dire. En résumé, le DNM fait bouger les esprits sur le dépistage prénatal généralisé, en premier lieu du côté des familles de malades dépistés, plus ponctuellement et indirectement semble-t-il du côté des cliniciens. Un autre épisode confirme ceci.

Le dernier volet de cette enquête s'établit non pas sur le lieu-même du CRCM, mais au niveau national. Il faut savoir que plusieurs programmes expérimentaux de dépistage généralisé de la mucoviscidose avant la naissance ont été effectués à partir des années 1990, notamment en Grande-Bretagne et aux Etats-Unis. Une tentative de ce type, visant à l'éradication de la mucoviscidose, a également été lancée au début des années 1990 en France, dans le Nord du Finistère, où son incidence est relativement élevée. Pour des raisons qui nous entraîneraient trop loin, essentiellement d'ordre technique et organisationnel, la tentative bretonne a tourné court. Or, en 2002, peu

${ }^{8}$ C'est moi qui traduis. 
après le lancement du DNM, un généticien moléculaire exerçant en province a proposé un nouveau programme pilote de dépistage prénatal généralisé de la mucoviscidose devant le Comité consultatif national d'éthique (CCNE), dont la mission est de donner des avis sur les problèmes éthiques soulevés par la biomédecine. Son idée consistait à effectuer un dépistage de la mutation la plus fréquente du gène impliqué dans la mucoviscidose chez toutes les femmes enceintes. L'objectif était de repérer les femmes hétérozygotes, après recueil de leur consentement, de façon à proposer aux futurs pères concernés une recherche de mutations et un éventuel test prénatal sur le fotus lorsque les deux parents étaient hétérozygotes. Lors de son lancement, ce généticien moléculaire a contesté l'efficacité du DNM, considérant qu'il ne permettait de gagner que quelques mois au moment du diagnostic et ne changeait pour ainsi dire rien au suivi thérapeutique. Il précise en entretien : «Vous savez, le nombre d'enfants qu'on reçoit dans le laboratoire qui sont dépistés en néonatal et [dont] on se dit : 'Cet enfant-là, on n'a pas donné la chance à ses parents de prendre une décision.' C'est troublant. » Il justifie sa proposition en ajoutant : «Je vous promets, c'est pas drôle un enfant muco. » Ainsi, l'idée du dépistage prénatal est apparue suite au dépistage néonatal et en lien avec celui-ci. En outre, dans le texte de proposition de programme pilote [2002], il met en exergue notamment «l'absence de traitement efficace de la maladie et les conditions de vie extrêmement difficiles ». Au total, il s'agit bien de qualifier la vie, car l'amélioration des soins ne conduit pas selon lui à une vie de qualité pour les enfants malades. D'ailleurs, dans le texte, il n'est plus question de vie des malades, mais de survie des enfants après le DNM.

\section{Les « pratiques » éthiques}

Les principaux responsables de l'AFDPHE sont opposés à cette politique de dépistage généralisé avant la naissance. Leurs arguments sont résumés dans un texte rédigé à l'occasion de l'audition de l'une de ces responsables par le CCNE lors de sa saisine. Ils sont de plusieurs ordres : médicaux (la maladie n'est pas suffisamment grave pour justifier cette action, alors qu'il existe un dépistage néonatal pour la prendre en charge), techniques (le test est proposé trop tardivement pendant la grossesse, le nombre de faux positifs et de faux négatifs attendus est trop important), économiques (le dépistage, l'information et les consultations associées sont trop coûteux) et moraux (d'une part la suppression de tous les futurs malades pour éradiquer une maladie pose un problème éthique, d'autre part, pour des raisons techniques, certains couples risquant de donner naissance à un enfant malade seront alarmés sans qu'il soit possible de leur proposer un diagnostic prénatal). Ces différents arguments recoupent largement ceux mis en avant par le CCNE, qui a rendu un avis négatif en 2004 sur cette proposition, en lui donnant une dimension à la fois technique, organisationnelle, médicale, économique et éthique [CCNE, 2004]. Hormis les aspects techniques et organisationnels, la dimension éthique semble constituer un obstacle à la généralisation du dépistage prénatal. Autrement dit, le «régime d'accoutumance » [Koch et Stemerding, 
1994] du DNM, qui semble faciliter l'entrée en scène du dépistage prénatal généralisé suite au dépistage néonatal, est bridé par un problème d'acceptabilité morale. Il s'agit d'aller plus loin dans la compréhension de celui-ci.

L'argument éthique est explicité par l'un des principaux responsables de l'AFDPHE en entretien. Le caractère généralisé et selon lui faussement facultatif de cette approche le heurte, car il considère qu'à partir du moment où un test est proposé en routine à 800000 femmes par an (correspondant au nombre d'accouchements en France), il est difficile pour une femme de le refuser. En somme, les «sujets éthiques » [Dean, 1999], celles et ceux qui sont censés opérer un choix, y seraient remis en cause. Toutefois, ceci pourrait s'appliquer également au DNM qui, pour des raisons qui sortiraient du cadre de ce texte, nécessite un consentement écrit de tous les parents. En effet, des observations montrent les limites de ces procédures de consentement, dont ce responsable est conscient [Vailly et Ensellem, 2010]. Ce dont il est question dans ces propos touche en réalité à une autre dimension que celle du choix et se réfère à ce qui est considéré comme une élimination systématique. "Hitler supprimait tous ceux qui n'étaient pas grands aux yeux bleus et qui étaient juifs par ailleurs. Bon, là je supprime tout enfant qui va être mucoviscidosique », dit-il. La comparaison avec les politiques de l'eugénisme hitlérien donne un caractère polémique à la position et accentue sa dimension morale. Elle a tendance à écraser les différences entre des politiques particulièrement autoritaires et une norme : les politiques hitlériennes dans un cas, des consentements un peu formels de l'autre. Elle écrase également les différences entre les « substances éthiques » [Dean, 1999] : des enfants ou des adultes dans un cas, des fœtus dans l'autre. En tout état de cause, ce sont donc les moyens mis en œuvre pour parvenir à un objectif de vie de qualité - soigner versus «éliminer» - qui sont surtout au cœur du débat.

Pour bien saisir cette position, il est nécessaire d'opérer un détour par les pratiques et les fondements moraux de l'AFDPHE. Tout d'abord, les responsables de l'association ne sont pas opposés par principe au diagnostic prénatal : ils ont participé eux-mêmes à nombre de tels diagnostics et mis certains de ces tests au point au cours de leur activité professionnelle. Au cours de leur carrière, s'ils n'étaient (ne sont) pas favorables à l'interruption volontaire de grossesse hors raison médicale - comme c'était le cas de beaucoup de personnes en France dans les années 1970 -, ils n'étaient pas défavorables aux interruptions médicales de grossesse, ayant été eux-mêmes confrontés à des situations familiales difficiles, et ont évolué probablement avec la société. Ensuite, la notion de prévention périnatale, qui établit un pont entre le dépistage néonatal et le diagnostic prénatal, est au cœur de l'activité fondatrice de l'AFDPHE. "Conseil génétique, diagnostic prénatal et dépistage néonatal sont les bases de la prévention périnatale d'un certain nombre de handicaps de l'enfant », écrivaient les responsables de l'association dans les années 1980. Cela n'étonnera pas outre mesure le lecteur familier des notions de prévention primaire (éviter les causes de la maladie, ici le développement du fœetus malade), secondaire (prévenir les conséquences physiques de la maladie) et tertiaire (éviter le handicap social et les complications), évoqués dans les ouvrages de santé publique et de génétique. Les buts de l'association, à l'origine, 
étaient donc la prévention périnatale, jusqu'à ce que le passage à la nomenclature de l'Assurance maladie des diagnostics prénatals, au cours des années 1990, ne les aient faits sortir du champ de l'AFDPHE. Mais pour l'association, le pont entre les approches néonatales et prénatales s'établit à certaines conditions, car le principe de légitimité du test prénatal continue d'être celui des grossesses dites à risque : lorsque les familles connaissent déjà un enfant malade ou sont porteuses de mutations, ou encore lorsque des signes échographiques ou biochimiques indiquent un risque de maladie. Il apparaît que les techniques prénatales étendues à des populations sans cas index et passant d'emblée par l'analyse génétique sont associées à la notion d'eugénisme, une notion qui fait véritablement barrage.

Au-delà de l'AFDPHE, les statistiques nationales permettent de confirmer la mise en pratique de ces principes. Pour la mucoviscidose, en 2006, environ 650 diagnostics prénatals sur fœetus ont été effectués en France, avec environ 50 interruptions médicales de grossesse à la clé en raison de tests positifs, à comparer avec les 180 bébés malades nés. Parmi ces diagnostics, un tiers concerne des couples avec antécédent familial et les deux tiers concernent des grossesses avec des signes d'appel échographique sans antécédent familial [Agence de la biomédecine, 2007, p. 226]. En Bretagne, une étude montre une baisse d'incidence de la mucoviscidose liée au diagnostic prénatal (toutes techniques confondues) non négligeable, puisqu'elle est évaluée par des biologistes à environ 30\% en dix ans [Scotet, Audrézet, Roussey, Rault et coll., 2003]. En somme, ces pratiques de diagnostic prénatal sont considérées comme légitimes en France - pour l'AFDPHE et au-delà - d'une manière qui a des effets en termes de population, mais sous réserve de laisser passer une certaine proportion d'enfants malades. La "pratique éthique », autrement dit la finalité de l'action dans un cadre éthique, est liée dans le cas présent au problème de l'extension des pratiques génétiques à visée éradicatrice. La promotion du rôle de l'individu ou du « cas par cas », en matière de diagnostic prénatal, reste de mise, dans un mouvement d'individuation connu autant des sociologues de la biomédecine [Clarke, Mamo, Fisman, Shim et coll., 2003] que des historiens [Turner, 2000]. Mais cette position n'est que majoritaire et pas unanime parmi les personnels soignants. En effet, d'un autre côté, la logique préventive se poursuit, comme à travers la proposition de dépistage prénatal généralisé émanant du généticien de province en 2002 ou des professionnels bretons dans les années 1990. Après être remonté dans le temps jusqu'à la naissance, pour intervenir le plus précocement possible, finalement certains parents et quelques soignants passent le seuil de la naissance pour remonter jusqu'au fœtus. Une fois ce seuil franchi, une intervention la plus précoce possible est défendue également pendant la grossesse.

\section{Conclusion}

Selon l'hypothèse formulée en introduction, la vie de qualité pour une maladie telle que la mucoviscidose peut s'analyser à partir de l'articulation entre le dépistage néona- 
tal, les pratiques de soins associées et l'approche prénatale. Or, «un des caractères essentiels de la biopolitique moderne (qui connaîtra son paroxysme au $\mathrm{XX}^{\mathrm{e}}$ siècle) est qu'il lui faut redéfinir sans cesse dans la vie le seuil qui articule et sépare ce qui est dedans et ce qui est dehors », dit Giorgio Agamben [1997, p. 142]. Assurément, il existe un effet de seuil relatif à la naissance : l'enfant malade est médicalement pris en charge de façon très serrée, alors que le fœtus peut faire l'objet d'une interruption de grossesse, y compris très tardivement. Soulignons que si ce seuil présente un caractère d'évidence aujourd'hui, l'anthropologie du droit montre que cela n'a pas toujours été le cas [Cayla et Thomas, 2002]. Historiquement, la Déclaration des droits de l'Homme de 1789 a consacré la vie naturelle, le simple fait de la naissance, comme source et porteuse du droit. Comme le dit Giorgio Agamben [1997, p. 138], « les déclarations des droits de l'homme représentent la figure originelle de l'inscription de la vie naturelle dans l'ordre juridico-politique de l'État-nation. Cette vie nue naturelle qui était dans l'Ancien Régime politiquement insignifiante et appartenait à Dieu comme vie de la créature, et qui, dans le monde classique, se distinguait clairement (du moins en apparence) [...] de la vie politique [...], émerge désormais au premier plan dans la structure de l'État, et devient le fondement terrestre de sa légitimité et de sa souveraineté ». En bref, naissance, vie et droits coïncident à partir de cette période, ce qui renforce l'effet de seuil. Mais l'enquête montre qu'autour de cette naissance s'exprime une tension. Le premier volet de la tension repose sur le fait que ce seuil s'estompe, et ceci pour plusieurs raisons. L'une d'elles a trait au fait qu'il existe un lien temporel entre le fœutus de 8 mois et demi et le nouveau-né d'un mois, et tient donc au moment particulier auquel est effectué le dépistage néonatal. Cet effet de continuité est accentué par la proximité des techniques (recherche de mutations chez l'enfant ou le fœtus), par la notion de prévention (s'appliquant aussi bien aux situations prénatales que postnatales) et, plus fondamentalement, par le droit à la bonne santé (mobilisé dans les deux cas). A ceci se greffent les diagnostics prénatals lors de grossesses ultérieures au dépistage néonatal, la question des formes frontières de maladie, les quelques centaines d'hétérozygotes repérés à la naissance et une tendance à remonter en amont. Pour ces différentes raisons, même si les approches prénatales et néonatales sont prises en charge par des spécialités différentes avec des modes d'intervention bien sûr différents, il est difficilement tenable de les séparer totalement. Assurer une bonne santé consiste dans les deux cas à éviter une maladie grave en situation périnatale, en lien avec des familles qui, depuis le XVIII ${ }^{\mathrm{e}}$ siècle selon Michel Foucault [2001], à la fois produisent une descendance, mais aussi fabriquent dans les meilleures conditions un être humain parvenant à l'état de maturité. Dans le cas de la trisomie 21 aux EtatsUnis, les recherches de Ryana Rapp [2000] montrent tous les moyens mis en œuvre à la fois pour détecter la maladie avant la naissance et pour prendre en charge le bébé une fois né avec la maladie, étant donné les problèmes médicaux auxquels il est susceptible d'être confronté. Les familles sont sous le regard aussi bien des obstétriciens que des pédiatres, car la grossesse comme le bébé sont médicalisés. Or, ce que montre ici l'enquête, c'est qu'il n'y a pas simplement une avancée parallèle de différentes pratiques, mais un lien de causalité entre les deux. Il existe des situations où les logiques 
de sélection de plus en plus exigeantes des fœtus et les logiques de soins de plus en plus attentifs des malades convergent. La recherche de bonne santé et l'élimination de la mauvaise santé ne semblent pas se neutraliser ni même s'ajouter, mais se renforcer. Précisons toutefois que ceci n'exclut pas des situations d'autres maladies où les traitements sont si efficaces qu'ils rendent le diagnostic prénatal sans objet, ce qui n'est pas le cas le plus fréquent. Dans le cadre de la mucoviscidose, des professionnels défendent une position éthique lorsqu'ils font valoir leur volonté d'atténuation des souffrances pour promouvoir l'éradication de la maladie, et c'est d'ailleurs ce qui fait tout l'intérêt des observations concernant la vie des malades. La finalité, les «pratiques éthiques » sont toujours l'évitement de souffrance et la compassion, mais au-delà de ces valeurs partagées, les moyens, le «travail éthique » pour parvenir à cet évitement de la souffrance sont divers et parfois en opposition. En effet, une position alternative est défendue par d'autres professionnels qui véhiculent le second volet de la tension. Cette position est représentée par les responsables de l'AFDPHE (qui s'attachent à bien séparer le DNM du diagnostic ou du dépistage prénatal) et par les pédiatres et les généticiens rencontrés lors de l'enquête à l'hôpital (qui s'interrogent sur leurs propres pratiques tout en les développant). Le mode d'incitation à reconnaître ces obligations morales repose alors sur la déontologie professionnelle (le rôle du médecin est de soigner), le code (l'un des critères de légitimité du dépistage néonatal est d'apporter un bénéfice à l'enfant dépisté) et/ou la loi (la législation française encadre les tests génétiques). Certains y ajoutent des interrogations ou des accusations liées à l'« eugénisme ». Je ne rependrai pas à mon compte cette qualification, car je pense, à l'instar de Nikolas Rose [2007], que les politiques et les pratiques d'aujourd'hui sont suffisamment complexes et nouvelles pour mériter leur propre vocabulaire. Il s'agit ici, pour reprendre l'idée défendue par Michel Foucault [Dean, 1999, p. 43], d'approcher le présent sans tomber dans «des déclarations théâtrales selon lesquelles le moment que nous vivons est une période de perdition totale, un abysse de noirceur, ou un avenir triomphant », mais de l'aborder en disant que «c'est un temps comme un autre, ou plutôt un temps qui n'est jamais tout à fait comme un autre $»^{9}$. Ce que suggère l'enquête de terrain, c'est que cette tension semble se déplacer du côté d'une remontée vers l'amont, du fait de l'idée de précocité qui imprègne toute la biomédecine actuelle. L'avenir dira si cette observation est confirmée.

\section{BIBLIOGRAPHIE}

AgAmben G. (1997[1995]), Homo Sacer. Le pouvoir souverain et la vie nue, Seuil, Paris.

ARMSTRONG D., LILFORD R., OGDEN J., WeSSELY, S. (2007), « Health-related quality of life and the transformation of symptoms », Sociology of Health \& Illness, 29(4),

\footnotetext{
${ }^{9}$ C'est moi qui traduis.
} 
p. $570-583$.

BASZANGER I. (1986), «Les maladies chroniques et leur ordre négocié », Revue Française de Sociologie, 27(1), p. 3-27.

BECK S. et NIEWÖHNER, J. (2009), «Localizing Genetic Testing and Screening in Cyprus and Germany: contingencies, continuities, ordering effects and bio-cultural intimacy », in ATKINSON P., GLASNER P., LOCK M. (dir.), Handbook of Genetics and Society. Mapping the New Genomic Era, Routledge, London, p. 76-93.

CAROL A. (1995), Histoire de l'eugénisme en France. Les médecins et la procréation $X I X^{e}-X X^{e}$ siècle, Seuil, Paris.

Cayla O. et ThOMas Y. (2002), Du droit de ne pas naître. A propos de l'affaire Perruche, Gallimard, Paris.

Clarke A.E., Mamo L., Fisman J.R., Shim J.K., Fosket J.R. (2003), « Biomedicalization: technoscientific transformations of health, illness, and U.S. biomedicine », American Sociological Review, 68, p. 161-194.

DEAN M. (1999), Governmentality: power and rule in modern society, Sage, London/Thousan Oaks/New Delhi.

Ehrich K., Williams C., ScotT R., SANDall J., FARsides B. (2006), « Social welfare, genetic welfare? Boundary-work in the IVF/PDG clinic », Social Science \& Medicine, 63, p. 1213-1224.

Foucault M. (2001[1994]), Dits et écrits [1976-1988], Gallimard, Paris.

KAUFERT P.A. (2000), «Screening the body: the pap smear and the mammogram », in LOCK M., Young A., CAMBrosio A. (dir.), Living and working with the new medical technologies. Intersections of inquiry, Cambridge University Press, Cambridge, p. 165-183.

KELLY M.P. et FIELD D. (1996), « Medical sociology, chronic illness and the body », Sociology of Health and Illness, 18(2), p. 241-257.

KOCH L. et STEMERDING D. (1994), « The sociology of entrenchment: a cystic fibrosis test for everyone? », Social Science \& Medicine, 39(9), p. 1211-1220.

LANDSMAN G. (2003), «Emplotting children's lives: developmental delay vs. disability », Social Science \& Medicine, 56(9), p. 1947-1960.

LAURENT-SEGUIN, O. (1999), Un cri d'espoir. Le combat d'une mère contre la mucoviscidose, Le Pré aux Clercs, Paris.

LEPLEGE A. (2004), «Qualité de vie (Mesures de la)», in LECOURT D. (dir.), Dictionnaire de la pensée médicale, Presses Universitaires de France, Paris, p. 939-940.

PETERSEN A. (2006), " The best experts: the narratives of those who have a genetic condition », Social Science \& Medicine, 63(1), p. 32-42.

PRESS N. et BROWNER C.H. (1997), «Why women say yes to prenatal diagnosis », Social Science \& Medicine, 45(7), p. 979-989.

RAPP R. (2000), « Extra Chromosomes and blue tulips: medico-familial interpretations », in LOCK M., Young A., CAMBrosio A. (dir.), Living and working with the new medical technologies. Intersections of inquiry, Cambridge University Press, Cambridge, p. 184-208.

REMENNICK L. (2006), « The quest for the perfect baby: why do Israeli women seek prenatal genetic testing ? », Sociology of Health \& Illness, 28(1), p. 21-53.

Rose N. (2007), The politics of life itself. Biomedicine, power and subjectivity in the twenty-first century, Princeton University Press, Princeton. 
SCOTET V., AudrÉZet M.-P., Roussey M., Rault G., Blayau M., De BraeKeleer M., FÉREC C. (2003), «Impact of public health strategies on the birth prevalence of cystic fibrosis in Brittany, France », Human Genetics, 113, p. 280-285.

Strauss A., Corbin J., Fagerhaugh S., Glaser B.G., Maines D., SuCZeK B., WieNER C. (1984 [1975]), Chronic illness and the quality of life, The C. V. Mosby Company, St. Louis.

TIMMERMAns S. et HAAS S. (2008), « Towards a sociology of disease », Sociology of Health and Illness, 30(5), p. 659-676.

TURNER B.S. (2000), « Changing concepts of health and illness », in ALBRECHT G. L., FITZPATRICK R., SCRIMSHAW S. C. (dir.), The handbook of social studies in health and medicine, SAGE, London, Thousand Oaks, New Delhi, p. 9-23.

VAILLY J. (2004), «Une politique de santé “a priori”. Le dépistage néonatal de la mucoviscidose en Bretagne », Sciences Sociales et Santé, 22(4), p. 35-60.

VAILLY J. (2006), «Genetic screening as a technique of government: the case of neonatal screening for cystic fibrosis in France », Social Science \& Medicine, 63 (12), p. 3092-3101.

VAILLY J. (2008), « The expansion of abnormality and the biomedical norm: neonatal screening, prenatal diagnosis and cystic fibrosis in France », Social Science \& Medicine, 66(12), p. 2532-2543.

VAILLY J. et ENSELLEM C. (2010), « Informing populations, governing subjects: the practices of screening for a genetic disease in France », in WIESER B. et BERGER W. (dir.), Assessing life: on the organisation of genetic testing, Profil, München/Wien, 225-253.

\section{Documents}

AFDPHE (2001), « 3 jours, l'âge du dépistage », AFDPHE (Assurance maladie - Sécurité sociale, Ministère de l'emploi et de la solidarité), Paris.

AGENCE DE LA BIOMEDECINE (2007), «Rapport annuel. Bilan des activités », SaintDenis.

Assurance Maladie (2000), Dossier de Presse «Un dépistage systématique de la mucoviscidose », 7 décembre 2000, Paris.

CCNE (2004), Avis n ${ }^{\circ} 83$ « Le dépistage prénatal généralisé de la mucoviscidose ».

ONM (2002), « Rapport sur la situation de la mucoviscidose en 2000 », Observatoire national de la mucoviscidose (Institut national d'études démographiques, Vaincre la mucoviscidose), Paris.

PROPOSITION DE PROGRAMME PILOTE (2002), « Dépistage de la mutation F508del en début de deuxième trimestre de grossesse. Estimation du risque d'attendre un enfant atteint de mucoviscidose. Proposition d'une étude pilote au CHU de Poitiers ». 